This is the final peer-reviewed accepted manuscript of:

Tralli, G., Uguzzoni, F. On a non-smooth potential analysis for Hörmander-type operators. Calc. Var. 57, 37 (2018).

The final published version is available online at: https://doi.org/10.1007/s00526018-1301-6

Rights / License:

The terms and conditions for the reuse of this version of the manuscript are specified in the publishing policy. For all terms of use and more information see the publisher's website. 


\title{
ON A NON-SMOOTH POTENTIAL ANALYSIS FOR HÖRMANDER-TYPE OPERATORS
}

\author{
GIULIO TRALLI AND FRANCESCO UGUZZONI
}

\begin{abstract}
We develop a potential theory approach for some degenerate parabolic operators in non-divergence form and with non-smooth coefficients, which are modeled on smooth Hörmander vector fields. We prove necessary and sufficient Wiener-type tests for the regularity of boundary points. As a consequence we obtain, in particular, a conetype criterion. We also investigate the related boundary value problem and the Hölder regularity at the boundary.
\end{abstract}

\section{INTRODUCTION}

Let $X_{1}, X_{2}, \ldots, X_{p}$ be a system of real smooth vector fields which are defined in some bounded open set $D_{0} \subset \mathbb{R}^{N}$, and satisfy the Hörmander's condition in $D_{0}$, i.e.

$$
\operatorname{rank} \operatorname{Lie}\left\{X_{1}, \ldots, X_{p}\right\}(x)=N \quad \text { at every point } x \in D_{0} .
$$

Denote by $d$ the related Carnot-Carathéodory control distance. Let us also fix $D$ a bounded open set compactly contained in $D_{0}$, and $-\infty<T_{1}<T_{2}<+\infty$. We want to consider the family of partial differential operators in the form

$$
\left.\mathcal{H}=\sum_{i, j=1}^{p} a_{i, j}(z) X_{i} X_{j}+\sum_{j=1}^{p} b_{j}(z) X_{j}-\partial_{t}, \quad \text { for } z=(x, t) \in D \times\right] T_{1}, T_{2}[
$$

where $a_{i, j}, b_{j}$ are $d$-Hölder continuous functions in $\left.D_{0} \times\right] T_{1}, T_{2}\left[\right.$, and the matrix $\left(a_{i, j}\right)_{i, j}$ is symmetric and uniformly positive definite.

Hörmander-type operators arise in many theoretical and applied settings sharing a subRiemannian underlying geometry, for instance in mathematical models for finance, control theory, geometric measure theory, pseudohermitian and CR geometry (see, e.g., [29, 1, 16, 9, 33, 28, 34; see also [8] and the references therein).

We want to study the Cauchy-Dirichlet problem associated with $\mathcal{H}$ with a potential theory approach. We are interested in regularity properties at the boundary for the generalized Perron-Wiener-Brelot-Bauer solutions (PW-solutions, in short) to the Dirichlet problem for $\mathcal{H}$ in generic bounded open sets $\Omega \subset \mathbb{R}^{N+1}$ compactly contained in $\left.D \times\right] T_{1}, T_{2}$ [. In particular, our aim is to prove necessary and sufficient conditions for the regularity of a boundary point in term of Wiener-type series, i.e. involving capacities of suitable compact sets which are ring-shaped with respect to $d$-Gaussians.

We recall that Wiener criteria are much more delicate for evolutive operators than for the stationary counterpart. Even for the heat operator, Wiener-type characterizations have quite a long history. To the best of our knowledge, the first attempt in this direction is due

2010 Mathematics Subject Classification. 35K65, 31E05, 31C15.

Key words and phrases. nondivergence Hörmander operators, potential analysis, boundary regularity. 
to Pini in [39] where he proved a sufficient condition in the 1-dimensional case for particular open sets. Then, in 32] Landis proved a characterization for the regularity in terms of a suitable series of caloric potentials. After the proof by Lanconelli of the necessary condition for the regularity in 24], the proper analogue of the classical Wiener criterion for the heat equation was finally proved by Evans and Gariepy in [14].

It is well-known that all the elliptic operators (with reasonable coefficients in the nondivergence case; see also 23 for recent developments) share the same regular points with the Laplacian, whereas Petrowski showed in [38] explicit counterexamples of this fact even for constant coefficients parabolic operators. This feature makes more interesting the study of the variable coefficients case. Several necessary and sufficient conditions have been investigated for classical parabolic operators both in divergence and non divergence form, also with different degree of regularity for the coefficients (see, e.g., the references [32, 37, 25, 26, 27]). The Evans-Gariepy Wiener test was extended to parabolic operators in divergence form with smooth variable coefficients by Garofalo and Lanconelli in [19], and with $C^{1}$-Dini continuous coefficients by Fabes-Garofalo-Lanconelli in [15].

In sub-Riemannian settings, as far as we know, the only Evans-Gariepy Wiener criterion is due to Garofalo and Segala in [20] for the heat equation on the Heisenberg group. We mention also the papers 40, 22 for Wiener test of Landis-type for a class of Kolmogorov equations. In these papers, the precise knowledge of the fundamental fundamental solution plays a crucial role. A different approach, inspired by [25, 26], has been carried out in [30] (see also [31, 42]) for a class of hypoelliptic diffusion operators. In [30] it is showed that Wiener-type tests can be derived starting just from the following key ingredient: Gaussian bounds of the fundamental solution with respect to a distance satisfying doubling condition and segment property. Let us mention more literature, always in settings of sub-Riemannian type, concerning the boundary behavior of nonnegative solutions to evolution equations in sufficiently regular domains (see, e.g., [10, 11, 17, 35] and the references therein).

Here we would like to pursue further the approach of 31, 30 by considering the case of the non-hypoelliptic operators in the non-divergence form 1.1. Even if the estimates in [31, 30] are basically independent of the smoothness of the coefficients, an approach based on the approximation of $\mathcal{H}$ via smooth operators seems to be not efficient with respect to the notions of PW-solution and of regularity of a boundary point (see Remark 2.1). Hence, we have to go through the classical axioms of potential theory for our non-smooth operator. Once we have in hands the notion of $\mathcal{H}$-regularity, we are going to prove the following

Theorem 1.1. Let $z_{0}=\left(x_{0}, t_{0}\right) \in \partial \Omega$, and $\left.\lambda \in\right] 0,1[$.

(i) If there exists $0<a \leq a_{0}$ and $b>b_{0}$ such that

$$
\sum_{h, k=1}^{+\infty} \frac{\mathcal{C}_{a}\left(\Omega_{k}^{h}\left(z_{0}, \lambda\right)\right)}{\left|B\left(x_{0}, \sqrt{\lambda^{k}}\right)\right|} \lambda^{b h}=+\infty
$$

then the point $z_{0}$ is $\mathcal{H}$-regular.

(ii) If the point $z_{0}$ is $\mathcal{H}$-regular, then

$$
\sum_{h, k=1}^{+\infty} \frac{\mathcal{C}_{b}\left(\Omega_{k}^{h}\left(z_{0}, \lambda\right)\right)}{\left|B\left(x_{0}, \sqrt{\lambda^{k}}\right)\right|} \lambda^{a h}=+\infty
$$

for every $b \geq \beta_{0}$ and $0<a \leq a_{0}$. 
The positive numbers $a_{0} \leq b_{0} \leq \beta_{0}$ are structural constants depending just on the vector fields $X_{1}, \ldots, X_{p}$, on the eigenvalue bounds for the matrix $\left(a_{i, j}\right)_{i, j}$, and on the Hölder norms of $a_{i, j}, b_{j}$.

Here $|E|$ denotes the Lebesgue measure of $E \subset \mathbb{R}^{N}, B\left(x_{0}, r\right)$ denotes the $d$-ball centered at $x_{0}$ of radius $r, \mathcal{C}_{a}$ stands for a suitable notion of capacity which is going to be stated precisely in subSection 3.2 and the compact sets $\Omega_{k}^{h}\left(z_{0}, \lambda\right)$ are defined in (4.1).

We remark that, as in [30, the sufficient condition $(1.2)$ and the necessary condition (1.3) are different. On the other hand, all the quantities and the objects appearing in our Wiener series depend on the underlying metric, and not on the fundamental solution of the specific operator $\mathcal{H}$. In fact, the lack of the precise behavior of the fundamental solution for Hörmander operators is one of the main obstacles for proving a characterization of EvansGariepy-Wiener type. Furthermore, with respect to the hypoelliptic case in [30, the presence of non-smooth coefficients forces us to follow a different approach for the proof of part (ii). As a consequence, the necessary condition 1.3 turns out to be slightly weaker in comparison with [30, Theorem 1.1]. We refer to Section 4 for the details of the technical reasons. We just mention here that this is due to the lack of suitable Riesz-type representations for $\mathcal{H}$ superharmonic functions, which indeed represents one of the main difficulties faced in this paper.

As a corollary of the above Theorem 1.1, we can show that a suitable notion of metric cone condition ensures the regularity of the boundary point. Moreover, the presence of such a cone condition allows us to derive Hölder estimates of the PW-solution $H_{\varphi}^{\Omega}$ up to the boundary.

Corollary 1.2. Assume $\Omega$ satisfies the exterior $d$-cone condition at $z_{0}$, according to Definition 4.2. Then

$$
z_{0} \text { is } \mathcal{H} \text {-regular. }
$$

If $\varphi \in C(\partial \Omega, \mathbb{R})$ satisfies in addition that $[\varphi]_{z_{0}, \delta}=\sup _{r>0} \sup _{\hat{d}\left(z, z_{0}\right) \leq r} \frac{\left|\varphi(z)-\varphi\left(z_{0}\right)\right|}{r^{\delta}}<\infty$ for some $\delta>0$, then there exist $0<\alpha_{0} \leq 1$ and $c>0$ such that

$$
\left|H_{\varphi}^{\Omega}(z)-\varphi\left(z_{0}\right)\right| \leq c[\varphi]_{z_{0}, \delta}\left(\hat{d}\left(z_{0}, z\right)\right)^{\alpha_{0}} \quad \text { for all } z \in \Omega \text {. }
$$

We can use the the PW-solution, together with fundamental solution of $\mathcal{H}$ constructed in [8, to actually solve the related boundary value problem in the following sense.

Corollary 1.3. Let $E$ be the set of points of $\partial \Omega$ such that 1.2 is satisfied for some $0<$ $a \leq a_{0}$ and $b>b_{0}$. Then, for every continuous datum $\varphi$ on $\partial \Omega$ and for every d-Hölder continuous function $f$ in a neighborhood of $\bar{\Omega}$, there exists

$$
u \in C(\Omega \cup E) \cap \mathcal{C}_{X}^{2}(\Omega) \text { such that } \quad \begin{cases}\mathcal{H} u=f & \text { in } \Omega, \\ u=\varphi & \text { on } E .\end{cases}
$$

In particular the above statement holds true even letting $E$ be the set of points of $\partial \Omega$ where the exterior d-cone condition is satisfied.

Remark 1.4. A weaker result than Corollary 1.3 was proved in [42. We stress that, from Corollary 1.3. we can deduce the existence of a unique solution to the Cauchy-Dirichlet problem related to $\mathcal{H}$ in the following cylindrical domains. Suppose $\Omega=A \times] t_{1}, t_{2}[$ is a bounded open set compactly contained in $D \times] T_{1}, T_{2}$ [ satisfying

$$
\forall x_{0} \in \partial A \quad \exists r_{0}, \theta_{0}>0 \text { such that }\left|B\left(x_{0}, r\right) \backslash A\right| \geq \theta_{0}\left|B\left(x_{0}, r\right)\right| \text { for all } 0<r \leq r_{0} .
$$


Then, for every continuous function $\varphi$ on $\partial_{p} \Omega$ and for every $d$-Hölder continuous function $f$ in a neighborhood of $\bar{\Omega}$, there exists a unique solution $u \in C\left(\Omega \cup \partial_{p} \Omega\right) \cap \mathcal{C}_{X}^{2}(\Omega)$ to the problem

$$
\begin{cases}\mathcal{H} u=f & \text { in } \Omega, \\ u=\varphi & \text { on } \partial_{p} \Omega .\end{cases}
$$

We will recall the classical definition of parabolic boundary $\partial_{p} \Omega$ in subSection 2.2.

The paper is organized as follows. In Section 2 we fix the main objects we are going to deal with and from which we can build the Potential Theory for our operators. In Section 3 we define the appropriate harmonic space of $\mathcal{C}_{X}^{2}$-functions, and we prove that suitable extensions of the operators $\mathcal{H}$ endow the strip $\left.\mathbb{R}^{N} \times\right] T_{1}, T_{2}$ [ with a structure of $\beta$-harmonic space satisfying the Doob convergence property. In Section 4 we finally prove Theorem 1.1. Corollary [1.2, and Corollary 1.3. In subSection 4.1, about the necessary condition for the regularity, we construct a special nonnegative hyperharmonic function by using a superposition of Riesz potentials with respect to a prototype operator, and then we invoke the classical potential theoretic notion of thinness. In subSection 4.2 for the sufficient condition and for the corollaries, we also show as intermediate steps an estimate of the modulus of continuity at the boundary for the PW-solution in Proposition 4.1, and a Hölder estimate of a suitable Wiener function in Proposition 4.3 under the $d$-cone condition.

\section{Preliminaries AND RECALLings}

Even though we want to study boundary value problems in bounded sets, our approach requires to exploit global objects. That is why we need to extend suitably the operator $\mathcal{H}$ in $\mathbb{R}^{N+1}$. This is possible thanks to the results in [8, Section 2-19]. In this section we are going to briefly recall the procedure. Then, we will introduce the main needed objects, namely the fundamental solution and the Green function for sets on which we can actually solve the Cauchy-Dirichlet problem.

2.1. Extension and global fundamental solution. It is possible to construct a system of smooth Hörmander vector fields $\tilde{X}_{1}, \tilde{X}_{2}, \ldots, \tilde{X}_{m}$ in $\mathbb{R}^{N}(m=p+N)$ coinciding in $D$ with the system $\left\{X_{1}, \ldots, X_{p}, 0, \ldots, 0\right\}$, and outside $D_{0}$ with the Euclidean system $\left\{0, \ldots, 0, \partial_{x_{1}}, \ldots, \partial_{x_{N}}\right\}$. Moreover, this can be done in such a way that the relative CarnotCarathéodory control distance $\tilde{d}$ satisfies:

(D1) the $\tilde{d}$-topology is the Euclidean topology, $\left(\mathbb{R}^{N}, \tilde{d}\right)$ is complete and, for every fixed $x \in \mathbb{R}^{N}, \tilde{d}(x, \xi) \rightarrow \infty$ if (and only if) $\xi \rightarrow \infty$ with respect to the usual Euclidean norm;

(D2) $\left(\mathbb{R}^{N}, \tilde{d}\right)$ is a doubling metric space w.r.t. the Lebesgue measure, i.e. there exists a constant $c_{\tilde{d}}>1$ such that

$$
|\tilde{B}(x, 2 r)| \leq c_{\tilde{d}}|\tilde{B}(x, r)|, \quad \forall x \in \mathbb{R}^{N}, \forall r>0 ;
$$

(D3) $\left(\mathbb{R}^{N}, \tilde{d}\right)$ has the segment property, i.e., for every $x, y \in \mathbb{R}^{N}$ there exists a continuous path $\gamma:[0,1] \rightarrow \mathbb{R}^{N}$ such that $\gamma(0)=x, \gamma(1)=y$ and

$$
\tilde{d}(x, y)=\tilde{d}(x, \gamma(t))+\tilde{d}(\gamma(t), y) \quad \forall t \in[0,1] .
$$


We stress that (D2) provides a global doubling condition for the extended vector fields, while general Hörmander vector fields satisfies usually just a local doubling condition thanks to the results in [36]. We also remark that any control distance satisfies the segment property (D3) (see, e.g., [13). Throughout the paper we will simply write $X_{1}, \ldots, X_{m}, d$ instead of $\tilde{X}_{1}, \ldots, \tilde{X}_{m}, \tilde{d}$. Let us also denote $\hat{d}\left((x, t),\left(x^{\prime}, t^{\prime}\right)\right)=\left(d\left(x, x^{\prime}\right)^{4}+\left(t-t^{\prime}\right)^{2}\right)^{\frac{1}{4}}$, and $\hat{B}(z, r)$ the relative parabolic balls.

If $E \subseteq \mathbb{R}^{N+1}$, we denote by $\mathcal{C}_{X}^{\beta}(E)$ the space of functions $u: E \rightarrow \mathbb{R}$ such that

$$
\|u\|_{\mathcal{C}_{X}^{\beta}(E)}:=\sup _{E}|u|+\sup _{z \neq z^{\prime} \in E} \frac{\left|u(z)-u\left(z^{\prime}\right)\right|}{\hat{d}\left(z, z^{\prime}\right)^{\beta}}<\infty .
$$

Moreover, $\mathcal{C}_{X}^{2+\beta}(E)$ denotes the space of functions $u: E \rightarrow \mathbb{R}$ which belong to $\mathcal{C}_{X}^{\beta}(E)$ together with any Lie-derivative along the vector fields $X_{1}, \ldots, X_{m}$ up to second order, and along $\partial_{t}$ up to first order. Analogously one defines $\mathcal{C}_{X, \text { loc }}^{2+\beta}(E)$.

Once we have extended the vector fields, we can also extend the operator. In fact, if we have

$$
\begin{aligned}
& \left.\Lambda^{-1}|\eta|^{2} \leq \sum_{i, j=1}^{p} a_{i, j}(z) \eta_{i} \eta_{j} \leq \Lambda|\eta|^{2} \quad \forall \eta \in \mathbb{R}^{p}, \forall z \in D_{0} \times\right] T_{1}, T_{2}[, \\
& \left\|a_{i, j}\right\|_{\mathcal{C}_{X}^{\alpha}\left(D_{0} \times\right] T_{1}, T_{2}[)},\left\|b_{j}\right\|_{\mathcal{C}_{X}^{\alpha}\left(D_{0} \times\right] T_{1}, T_{2}[)}, \leq k
\end{aligned}
$$

for some positive constants $k, \Lambda \geq 1$, and $\alpha \in(0,1)$, then we can extend the operator $\mathcal{H}$ in (1.1) to an operator

$$
\tilde{\mathcal{H}}=\sum_{i, j=1}^{m} \tilde{a}_{i, j}(z) X_{i} X_{j}+\sum_{j=1}^{m} \tilde{b}_{j}(z) X_{j}-\partial_{t}, \quad \text { for } z \in \mathbb{R}^{N+1},
$$

coinciding with $\mathcal{H}$ in $D \times] T_{1}, T_{2}$ [ and with the heat operator in $\left(\mathbb{R}^{N} \backslash D_{0}\right) \times \mathbb{R}$. Moreover the coefficients of $\tilde{\mathcal{H}}$ can be built such that

$$
\begin{aligned}
& \Lambda^{-1}|\eta|^{2} \leq \sum_{i, j=1}^{m} \tilde{a}_{i, j}(z) \eta_{i} \eta_{j} \leq \Lambda|\eta|^{2} \quad \forall \eta \in \mathbb{R}^{m}, \forall z \in \mathbb{R}^{N+1}, \\
& \left\|\tilde{a}_{i, j}\right\|_{\mathcal{C}_{X}^{\alpha}\left(\mathbb{R}^{N+1}\right)},\left\|\tilde{b}_{j}\right\|_{\mathcal{C}_{X}^{\alpha}\left(\mathbb{R}^{N+1}\right)}, \leq \tilde{k}=C k .
\end{aligned}
$$

Again, the will be always dropped. In [8, Theorem 10.7] it is proved that such extended operators have a global fundamental solution $\Gamma\left(\mathcal{C}_{X}^{2+\alpha}\right.$ out of the diagonal of $\left.\mathbb{R}^{N+1} \times \mathbb{R}^{N+1}\right)$ satisfying two sided Gaussian-type estimates on strips. Precisely, denoting the $d$-Gaussian (with exponent $a>0$ ) by

$$
\mathrm{G}_{a}(z, \zeta)=\mathrm{G}_{a}(x, t, \xi, \tau)= \begin{cases}0 & \text { if } t \leq \tau, \\ \frac{1}{|B(x, \sqrt{t-\tau})|} \exp \left(-a \frac{d^{2}(x, \xi)}{t-\tau}\right) & \text { if } t>\tau,\end{cases}
$$

we have the existence of positive constants $a_{0} \leq b_{0}$ and, for any open and bounded time interval $I$, the existence of $C_{0}=C_{0}(I)>0$ such that

$$
\frac{1}{C_{0}} \mathrm{G}_{b_{0}}(z, \zeta) \leq \Gamma(z, \zeta) \leq C_{0} \mathrm{G}_{a_{0}}(z, \zeta), \quad \forall z, \zeta \in \mathbb{R}^{N} \times I .
$$

The constants $a_{0}, b_{0}, C_{0}$ depend on the original vector fields, and on the constants $\Lambda, \alpha, k$ in (2.1) and (2.2). We denote by

$$
\left.S=\mathbb{R}^{N} \times\right] T_{1}, T_{2}[
$$


When we don't make further comments, the constant $C_{0}$ will stand for $C_{0}(] T_{1}, T_{2}[)$.

2.2. Solvability and Green functions. For the uniqueness of the solutions of the CauchyDirichlet problem, as well as for future applications, we are going to need the following maximum principle in the class $\mathcal{C}_{X}^{2}$ :

let $\Omega$ be an open set, whose closure is contained in a strip $\mathbb{R}^{N} \times I$. For a fixed $T \in I$ let us define $\Omega_{T}=\{(x, t) \in \Omega \mid t<T\}$ and $\partial_{T} \Omega=\{(x, t) \in \partial \Omega \mid t<T\}$. If $u \in \mathcal{C}_{X}^{2}(\Omega)$ satisfies

$$
\left\{\begin{array}{ll}
\mathcal{H} u \geq 0 & \text { in } \Omega_{T}, \\
\lim \sup _{\Omega_{T} \ni z \rightarrow \zeta} u(z) \leq 0 & \text { for every } \zeta \in \partial_{T} \Omega, \\
\lim \sup _{\Omega_{T} \ni z \rightarrow \infty} u(z) \leq 0 & \text { if } \Omega_{T} \text { is unbounded, }
\end{array} \quad \text { then } u \leq 0 \text { in } \Omega_{T} .\right.
$$

The scheme for proving such a maximum principle is classical. The non-classical difficulty relies on the regularity class $\mathcal{C}_{X}^{2}$ : we refer the reader to [8, Theorem 13.1] for the proof, which is based on the maxima propagation result for $\mathcal{C}_{X}^{2}$-functions proved in [4].

Let us now take a bounded open set $A \subset \mathbb{R}^{N}$, and consider the cylinder $\left.\mathfrak{C}=A \times\right] t_{1}, t_{2}[$ for some $t_{1}<t_{2}$. It is possible to define the parabolic boundary of a cylinder, namely

$$
\partial_{p} \mathfrak{C}=\left(\partial A \times\left[t_{1}, t_{2}\right]\right) \cup\left(\bar{A} \times\left\{t_{1}\right\}\right) .
$$

With these notations, we shall say that $\mathfrak{C}$ is $\mathcal{H}$-solvable if, for every continuous function $\varphi$ on $\partial_{p} \mathfrak{C}$, there exists a solution $u=u_{\varphi}$ to

$$
u \in \mathcal{C}_{X}^{2}(\mathfrak{C}) \cap C\left(\mathfrak{C} \cup \partial_{p} \mathfrak{C}\right), \quad \mathcal{H} u=0 \quad \text { in } \mathfrak{C}, \quad u=\varphi \quad \text { on } \partial_{p} \mathfrak{C}
$$

Such a solution, if it exists, has to be unique by the aforementioned maximum principle. We also denote $A \subset \mathbb{R}^{N}$ to be $\mathcal{H}$-solvable if, for every $t_{1}<t_{2}$, the cylinder $\left.A \times\right] t_{1}, t_{2}[$ is $\mathcal{H}$-solvable.

In [42, Theorem 4.1] it has been proved that $\mathfrak{C}$ is $\mathcal{H}$-solvable if any point of $\partial_{p} \mathfrak{C}$ satisfies an exterior $d$-cone condition, i.e. if, for any $\left(x_{0}, t_{0}\right) \in \partial_{p} \mathfrak{C}$, there exist $M_{0}, r_{0}, \theta_{0}>0$ such that

$$
\left|\left\{x \in \overline{B\left(x_{0}, M_{0} r\right)}:\left(x, t_{0}-r^{2}\right) \notin \mathfrak{C}\right\}\right| \geq \theta_{0}\left|B\left(x_{0}, M_{0} r\right)\right| \quad \text { for all } 0<r \leq r_{0} .
$$

The proof is based on a regularization technique for the operator coefficients (see the details in [8, Section 14]), on interior Schauder estimates [7, and on careful uniform estimates at the boundary for the solutions of smooth operators under condition 2.5 (see also [31, Theorem $9.1])$.

Remark 2.1. We would like to comment on the approach we have just mentioned. A suitable regularization for the operator and an approximation procedure via the solutions of the related smooth operators are powerful tools. One can thus obtain a solution for the non-smooth operator as limit of solutions, and also regularity at the boundary if the boundary estimates are stable in the approximation. On the other hand, as we will see in the next section, we can develop a Potential Theory for our non-smooth operator $\mathcal{H}$. As a consequence, we will have the notion of generalized $P W$-solution and of $\mathcal{H}$-regularity in the classical Perron-Wiener sense. It seems to be troublesome to understand whether such a $P W$-solution appears as the limit of a sequence of approximated $P W$-solutions, and also whether the $\mathcal{H}$-regularity of a boundary point says something about the behavior of this sequence. Therefore, we will deal with the proof of Theorem 1.1 with a direct approach, by working directly with the non-smooth operator $\mathcal{H}$. 
There exist bounded open sets $A \subset \mathbb{R}^{N}$ such that $\left.A \times\right] t_{1}, t_{2}[$ satisfies condition (2.5) at any parabolic boundary point and for any $t_{1}<t_{2}$, which says in our notations that there exist $\mathcal{H}$-solvable sets. It is enough (see [31, Proposition 6.1]) that, for every $x_{0} \in \partial A$, there exist $r_{0}, \theta_{0}>0$ such that

$$
\left|B\left(x_{0}, r\right) \backslash A\right| \geq \theta_{0}\left|B\left(x_{0}, r\right)\right| \quad \text { for all } 0<r \leq r_{0} .
$$

Under this condition, the set $A$ is $\mathcal{H}$-solvable. There are, actually, plenty of sets fulfilling this requirement. As a matter of fact, for any bounded open set $B \subset \mathbb{R}^{N}$ and for every $\delta>0$ there exist two $\mathcal{H}$-solvable sets $A_{\delta}, A^{\delta}$ such that

$$
\{x \in B: d(x, \partial B)>\delta\} \subseteq A_{\delta} \subseteq B \subseteq A^{\delta} \subseteq\left\{x \in \mathbb{R}^{N}: d(x, \bar{B})<\delta\right\} .
$$

Following [31, Theorem 6.5], the idea for the proof is to cut away from $B$ small metric balls centered on $\partial B$.

Proceeding as in 8, Theorem 16.3 and Lemma 16.4] (see also [5]), we have the existence of a Green function $G=G^{A}$ related to $\mathcal{H}$-solvable sets $A$. More precisely, $G$ is a continuous function on the set $\{(z, \zeta) \in(\bar{A} \times \mathbb{R}) \times(A \times \mathbb{R}): z \neq \zeta\}$, and it satisfies $0 \leq G \leq \Gamma$. Moreover, for every fixed $\zeta \in A \times \mathbb{R}$, the function $G(\cdot, \zeta) \in \mathcal{C}_{X}^{2}(A \times \mathbb{R} \backslash\{\zeta\})$ solves $\mathcal{H}(G(\cdot, \zeta))=0$ in $A \times \mathbb{R} \backslash\{\zeta\}, G(\cdot, \zeta)=0$ in $\partial A \times \mathbb{R}$. Also, for every $\varphi \in C(\bar{A})$ such that $\varphi=0$ in $\partial A$, and for every fixed $\tau \in \mathbb{R}$, the function

$$
u(x, t)=\int_{A} G(x, t, \xi, \tau) \varphi(\xi) \mathrm{d} \xi, \quad x \in \bar{A}, t>\tau,
$$

is continuous on $\bar{A} \times[\tau,+\infty[$ and it solves

$$
\mathcal{H} u=0 \text { in } A \times] \tau,+\infty[, \quad u=0 \text { in } \partial A \times[\tau,+\infty[, \quad u(\cdot, \tau)=\varphi \text { in } \bar{A} .
$$

In the proof of part (i) of Theorem 1.1 it will be important to derive precise estimates for the Green functions of $\mathcal{H}$-solvable sets scaling accordingly to the metric $d$. Let us fix now these sets. Thanks to (2.7), for every $z_{0} \in \mathbb{R}^{N+1}$ and for every $0<r$ there exists a $\mathcal{H}$-solvable set $D\left(x_{0}, r\right)$ satisfying $B\left(x_{0}, \frac{1}{2} r\right) \subseteq D\left(x_{0}, r\right) \subseteq B\left(x_{0}, r\right)$. For every $M \geq e$ and $r>0$ small enough, we fix

$$
\left.C_{z_{0}}(M, r)=D\left(x_{0}, \sqrt{r \log (M)}\right) \times\right] t_{0}-r, t_{0}+r[
$$

and we denote by $G\left(z_{0}, M, r ; z, \zeta\right)$ the Green function of $D\left(x_{0}, \sqrt{r \log (M)}\right)$ restricted to $C_{z_{0}}(M, r)$.

\section{Potential theory for $\mathcal{H}$}

In this section we show that $\mathcal{H}$ endows the strip $\left.S=\mathbb{R}^{N} \times\right] T_{1}, T_{2}[$ with a structure of $\beta$ harmonic space. The potential theory we want to exploit has to deal with the $\mathcal{C}_{X}^{2}$-functions. Given an open set $\Omega \subset S$ we denote by $\mathrm{H}(\Omega)$ the family of the $\mathcal{H}$-harmonic functions on $\Omega$. For our purposes this is given by

$$
\mathrm{H}(\Omega)=\left\{u \in \mathcal{C}_{X}^{2}(\Omega) \mid \mathcal{H} u=0 \text { in } \Omega\right\} .
$$

Clearly $\Omega \mapsto \mathrm{H}(\Omega)$ is a sheaf of functions on $S$, which is harmonic since $\mathrm{H}(\Omega)$ is a linear subspace of $C(\Omega)$ (hereafter we use the standard notation of Potential Theory in Abstract Harmonic Spaces, as presented, e.g., in [12]). 
A bounded open set $V \subset S$ is called $\mathcal{H}$-regular if, for every continuous function $\varphi: \partial V \rightarrow \mathbb{R}$, there exists a function $u: V \rightarrow \mathbb{R}, \mathcal{H}$-harmonic in $V$ and such that

$$
\lim _{V \ni x \rightarrow y} u(x)=\varphi(y) \quad \text { for every } y \in \partial V \text {. }
$$

Thanks to the Maximum Principle in 2.4), this function $u$ is unique, and nonnegative whether $\varphi \geq 0$. It will be denoted by $H_{\varphi}^{V}$. Then, if $V$ is an open $\mathcal{H}$-regular set and $z \in V$, the map

$$
\varphi \mapsto H_{\varphi}^{V}(z)
$$

defines a linear and positive functional on $C(\partial V)$. As a consequence, there exists a nonnegative Radon measure $\mu_{z}^{V}$ supported on $\partial V$ such that

$$
H_{\varphi}^{V}(z)=\int_{\partial V} \varphi(\zeta) \mathrm{d} \mu_{z}^{V}(\zeta) \quad \forall \varphi \in C(\partial V) .
$$

We call $\mu_{z}^{V}$ the $\mathcal{H}$-harmonic measure related to $V$ and $z$. A function $\left.\left.u: \Omega \rightarrow\right]-\infty, \infty\right]$ will be called $\mathcal{H}$-hyperharmonic in $\Omega$ if $u$ is lower semi-continuous and

$$
u(z) \geq \int_{\partial V} u(\zeta) \mathrm{d} \mu_{z}^{V}(\zeta) \quad \forall z \in V,
$$

for every $\mathcal{H}$-regular open set $V \subset \bar{V} \subseteq \Omega$. The last condition is equivalent to ask the following

$$
H_{\varphi}^{V} \leq u \text { in } V \forall \varphi \in C(\partial V) \text { with } \varphi \leq\left. u\right|_{\partial V} .
$$

We shall denote by $\mathrm{H}^{*}(\Omega)$ the family of the $\mathcal{H}$-hyperharmonic functions in $\Omega$. If $u \in \mathrm{H}^{*}(\Omega)$ and $u<+\infty$ in a dense subset of $\Omega$, then we say that $u$ is $\mathcal{H}$-superharmonic in $\Omega$ and we write $u \in \overline{\mathrm{H}}(\Omega)$.

In order to have the structure of $\beta$-harmonic space, we need some axioms to be satisfied. The axiom of positivity is trivial for us since the function $u \equiv 1$ is $\mathcal{H}$-harmonic and strictly positive in $S$.

The axiom of separation can be proved as in [31, Proposition 3.6], by using the presence of the fundamental solution $\Gamma$. The fact that, for any fixed $\zeta \in S, \Gamma(\cdot, \zeta) \in C_{X}^{2}(S \backslash\{\zeta\})$ is a $\mathcal{H}$-superharmonic function (actually a $\mathcal{H}$-potential) can be proved as in [31, Proposition 3.4]. Also the Green function $G(\cdot, \zeta)$ related to a $H$-solvable set is then $\mathcal{H}$-superharmonic. Moreover, if $\nu$ is a nonnegative Radon measure with compact support, we have

$$
\Gamma * \nu(\cdot):=\int_{S} \Gamma(\cdot, \zeta) \mathrm{d} \nu(\zeta) \in \mathrm{H}^{*}(S) .
$$

As a matter of fact, the lower semicontinuity comes just from Fatou lemma and, since $\Gamma(\cdot, \zeta) \in \overline{\mathrm{H}}(S)$, we also get from Tonelli theorem that

$$
\Gamma * \nu(z)=\int_{S} \Gamma(z, \zeta) \mathrm{d} \nu(\zeta) \geq \int_{S} \int_{\partial V} \Gamma\left(\zeta^{\prime}, \zeta\right) \mathrm{d} \mu_{z}^{V}\left(\zeta^{\prime}\right) \mathrm{d} \nu(\zeta)=\int_{\partial V} \Gamma * \nu\left(\zeta^{\prime}\right) \mathrm{d} \mu_{z}^{V}\left(\zeta^{\prime}\right)
$$

for all $z \in V$, and for all $\mathcal{H}$-regular open sets $V \subset \bar{V} \subseteq S$. The fact that Riesz-type functions as $\Gamma * \nu$ are $\mathrm{H}^{*}$-functions will be crucial for us in the proof of Theorem 1.1 in Section 4 .

For the axiom of convergence in the sense of Doob, we have to prove that the limit of any increasing sequence of $\mathcal{H}$-harmonic functions in an open set $O \subseteq S$ is $\mathcal{H}$-harmonic whenever it is finite in a dense subset of $O$. It is well-known that this can be proved straightforwardly once one has in hands a parabolic Harnack inequality. For our non-smooth operators (1.1) the validity of suitable scale invariant Harnack inequalities has been proved in [8, Theorem 
15.1], as an ending point of a series of investigations [2, 3, 5, 7, 42, 31] (and references therein).

The last axiom we have to satisfy is the so-called axiom of regularity: we need that the family of $\mathcal{H}$-regular subsets of $S$ forms a basis for the Euclidean topology. In the case of smooth coefficients, the hypoellipticity and the non-totally degeneracy of the Hörmandertype operators allow to trigger the procedure by Bony in [6. Since we want to deal with operators with non-smooth coefficients, we are going to argue carefully by regularization (the same regularization procedure we have briefly mentioned before Remark 2.1). We are now going to show this fact in details. To this purpose let us consider, for $\varepsilon>0$, suitable regularized operators

$$
\mathcal{H}^{\varepsilon}=\sum_{i, j=1}^{m} a_{i, j}^{\varepsilon}(x, t) X_{i} X_{j}+\sum_{k=1}^{m} b_{k}^{\varepsilon}(x, t) X_{k}-\partial_{t},
$$

where $a_{i, j}^{\varepsilon}, b_{k}^{\varepsilon}$ are smooth and uniformly convergent on compact sets to $a_{i, j}, b_{k}$, as $\varepsilon \rightarrow 0^{+}$. This regularization can be constructed in such a way that the $\mathcal{C}_{X}^{\alpha}$-norm of $a_{i, j}^{\varepsilon}, b_{k}^{\varepsilon}$ is taken under control as $\varepsilon \rightarrow 0^{+}$(as well as the ellipticity constants of $a_{i, j}^{\varepsilon}$ ): this is proved in 8, Theorem 14.2] via an ad hoc mollification, i.e. a convolution with the fundamental solution of $\sum X_{j}^{2}-\partial_{t}$. Fix now a point $z_{0}=\left(x_{0}, t_{0}\right) \in S$. If we denote the vector fields $X_{j}=$ $\sum_{k} \sigma_{j, k}(x) \partial_{x_{k}}$, the Hörmander condition implies in particular that the matrix $\sigma^{t}\left(x_{0}\right) \sigma\left(x_{0}\right)$ cannot be the zero matrix. Without loss of generality, we can think $\left(\sigma^{t}\left(x_{0}\right) \sigma\left(x_{0}\right)\right)_{1,1}>0$. We can consider, by continuity and by uniform convergence, an open neighborhood $U$ of $z_{0}$ where

$$
\inf _{z=(x, t) \in U}\left(\sigma^{t}(x) A^{\varepsilon}(z) \sigma(x)\right)_{1,1} \geq \frac{1}{2 \Lambda} \inf _{(x, t) \in U}\left(\sigma^{t}(x) \sigma(x)\right)_{1,1} \geq \frac{1}{4 \Lambda}\left(\sigma^{t}\left(x_{0}\right) \sigma\left(x_{0}\right)\right)_{1,1}
$$

for all $\varepsilon$. In $U$ we can construct a basis of neighborhoods of $z_{0}$ as in [6], i.e. bounded sets with Lipschitz boundary as $V_{\rho, M}=B\left(\left(x_{0}+M e_{1}, t_{0}\right), M+\rho\right) \cap B\left(\left(x_{0}-M e_{1}, t_{0}\right), M+\rho\right)$ (for suitable $M, \rho>0$ ). For any $\varepsilon>0$, the Dirichlet problem

$$
\begin{cases}\mathcal{H}^{\varepsilon} v=0 & \text { in } V_{\rho, M}, \\ v=\varphi & \text { on } \partial V_{\rho, M}\end{cases}
$$

can be classically solved for any $\varphi \in C\left(\partial V_{\rho, M}\right)$. In fact, the hypoellipticity of the operators $\mathcal{H}^{\varepsilon}$ allows to solve such problem via elliptic regularization and the exterior ball property of the sets $V_{\rho, M}$ allows to build $\mathcal{H}^{\varepsilon}$-barrier functions which guarantee the continuity up to the boundary of the solutions (see, e.g, 6. Théorème 5.2]). Let us call $u_{\varepsilon}$ the unique solutions of such problems. The family $\left(u_{\varepsilon}\right)_{\varepsilon}$ is equibounded by $\max _{\partial V_{\rho, M}}|\varphi|$. Moreover, by 7$]$ we have the interior Schauder estimates

$$
\left\|u_{\varepsilon}\right\|_{\mathcal{C}_{X}^{2, \alpha}(K)} \leq C\left\|u_{\varepsilon}\right\|_{\infty}
$$

in any compact set $K \subset V_{\rho, M}$, with $C$ independent of $\varepsilon$ (it depends just on the ellipticity constants, and the $d$-Hölder modulus of the operator coefficients). Thus, $u_{\varepsilon}$ converges to a function $u$ in $\mathcal{C}_{X}^{2}$, as $\varepsilon \rightarrow 0^{+}$. In particular $u \in \mathrm{H}\left(V_{\rho, M}\right)$. In order to say that $V_{\rho, M}$ are $\mathcal{H}$-regular, we have to prove that $u$ is continuous up to the boundary and it attains there the datum $\varphi$. It is enough to see that, for any point $\zeta \in \partial V_{\rho, M}$ the modulus of continuity of $u_{\varepsilon}$ at $\zeta$ is independent of $\varepsilon$. This is possible since we can find $\mathcal{H}^{\varepsilon}$-barrier functions which 
are independent of $\varepsilon$. More precisely, there exist $M_{0}, \rho_{0}$ such that, for every $M \geq M_{0}$ and $\rho \leq \rho_{0}$ and for every point $\zeta=(y, s)$, the Hopf-type barrier

$$
h(x, t)=e^{-C r^{2}}-e^{-C\left(\left\|x-\xi_{0}\right\|^{2}+\left(t-\tau_{0}\right)^{2}\right)}
$$

is a $\mathcal{H}^{\varepsilon}$-barrier for all $\varepsilon>0$. Here $r^{2}=\left\|y-\xi_{0}\right\|^{2}+\left(s-\tau_{0}\right)^{2}$, the point $\left(\xi_{0}, \tau_{0}\right)$ is such that $\overline{B\left(\left(\xi_{0}, \tau_{0}\right), r\right)} \backslash\{\zeta\} \cap \overline{V_{\rho, M}}=\emptyset$. In order to check this is an actual barrier function (that is $\mathcal{H}^{\varepsilon} h \leq 0$ in an open neighborhood $U_{\zeta}$ of $\left.\zeta\right)$ we have to exploit $(3.2)$ and the definition of $V_{\rho, M}$. The constant $C$ and the neighborhood $U_{\zeta}$ depend on the smoothness of the vector fields, on $\Lambda$, and on the boundedness of $b_{j}$ (but not on $\varepsilon$ ). This concludes the proof that $\left(V_{\rho, M}\right)_{\rho \leq \rho_{0}, M \geq M_{0}}$ is a $\mathcal{H}$-regular basis of neighborhoods of $z_{0}$.

3.1. Potential theory tools. We have thus proved that the operator $\mathcal{H}$ endows the strip $S$ with a structure of $\beta$-harmonic space satisfying the Doob convergence property. As a consequence, for any bounded open set $\Omega$ with $\bar{\Omega} \subseteq S$, the Dirichlet problem

$$
\left\{\begin{array}{l}
\mathcal{H} u=0 \text { in } \Omega \\
\left.u\right|_{\partial \Omega}=\varphi
\end{array}\right.
$$

has a generalized solution $H_{\varphi}^{\Omega}$, in the Perron-Wiener sense, for every continuous function $\varphi: \partial \Omega \rightarrow \mathbb{R}$. Therefore we can define the notion of regularity for a boundary point. A point

$$
\begin{gathered}
z_{0} \in \partial \Omega \text { is called } \mathcal{H} \text {-regular if } \\
\lim _{\Omega \ni z \rightarrow z_{0}} H_{\varphi}^{\Omega}(z)=\varphi\left(z_{0}\right) \quad \text { for every } \varphi \in C(\partial \Omega) .
\end{gathered}
$$

Classical characterizations for the regularity are available in $\beta$-harmonic spaces. Some of them are related with the notion of balayage. We recall the definition for the convenience of the reader. In what follows we agree to let $\liminf _{\zeta \rightarrow z} w(\zeta)=\sup _{V \in \mathcal{U}_{z}}\left(\inf _{V} w\right)$ being $\mathcal{U}_{z}$ a basis of neighborhoods of $z$.

Definition 3.1. Given a nonnegative $\mathcal{H}$-hyperharmonic function $u_{0}$ in $S$, and a subset $E$ of $S$, we denote the $\mathcal{H}$-reduced function of $u_{0}$ on $E$ as

$$
R_{u_{0}}^{E}=\inf \left\{v: v \in \overline{\mathrm{H}}(S), v \geq 0 \text { in } S, v \geq u_{0} \text { in } E\right\} .
$$

We can thus define the $\mathcal{H}$-balayage of $u_{0}$ on $E$ as

$$
\widehat{R}_{u_{0}}^{E}(z)=\liminf _{\zeta \rightarrow z} R_{u_{0}}^{E}(\zeta), \quad z \in S .
$$

We denote by ${ }^{U_{0}} \widehat{R}_{u_{0}}^{E}$ the balayage with respect to the harmonic space restricted to $U_{0}$.

It will be important for us (see 4.9 below) to control the modulus of continuity of the PW-solution in terms of a series of balayage functions $\widehat{R}_{1}^{K_{n}}$ for suitable compact sets $K_{n}$ (see also [31, Theorem 5.2]). Moreover, it will be crucial the classical notion of thinness (see, e.g., [12, pg. 149]) in the proof of part (ii) of Theorem 1.1 .

Definition 3.2. A set $E \subset S$ is said to be $\mathcal{H}$-thin at $\zeta_{0} \in S$ if there exist two open neighborhoods $V_{0} \subset U_{0}$ of $\zeta_{0}$ and a nonnegative $\mathcal{H}$-hyperharmonic function $u_{0}$ on $U_{0}$ such that

$$
{ }^{U_{0}} \widehat{R}_{u_{0}}^{E \cap V_{0}}\left(\zeta_{0}\right)<u_{0}\left(\zeta_{0}\right) .
$$


Since we are in a $\beta$-harmonic space, we can always consider $u_{0} \equiv 1$ in the previous definition (see [12, Proposition 6.3.2]). Moreover, the following characterization holds true in $\beta$-harmonic spaces by [12, Theorem 6.3.3]:

$$
z_{0} \in \partial \Omega \text { is } \mathcal{H} \text {-regular if and only if } S \backslash \Omega \text { is not } \mathcal{H} \text {-thin at } z_{0} .
$$

The fact we are dealing with evolutive operators allows us the see that $\mathbb{R}^{N} \times\left\{t \geq t_{0}\right\}$ is always $\mathcal{H}$-thin at points $z_{0}=\left(x_{0}, t_{0}\right)$. As a matter of fact, if we denote $w=\inf _{\varepsilon>0} \chi_{\left\{t>t_{0}-\varepsilon\right\}}$ and we notice that the characteristic functions $\chi_{\left\{t>t_{0}-\varepsilon\right\}}$ are $\mathcal{H}$-superharmonic by the maximum principle in 2.4, we have

$$
\hat{R}_{1}^{\mathbb{R}^{N} \times\left\{t \geq t_{0}\right\}}\left(z_{0}\right) \leq \liminf _{z \rightarrow z_{0}} w(z)=0<1 .
$$

Hence, since the union of two sets which are thin at $z_{0}$ is thin at $z_{0}$ by [12, Theorem 6.3.1], we have that

$$
(S \backslash \Omega) \cap\left\{t<t_{0}\right\} \text { is } \mathcal{H} \text {-thin at } z_{0} \quad \Longrightarrow \quad S \backslash \Omega \text { is } \mathcal{H} \text {-thin at } z_{0} .
$$

3.2. Gaussian capacities. We also fix the definition of capacity $\mathcal{C}_{a}$ with respect to the Gaussian $\mathrm{G}_{a}$. Let us denote by $\mathcal{F}\left(\mathbb{R}^{N+1}\right)$ the collection of the compact subsets of $\mathbb{R}^{N+1}$. For $F \in \mathcal{F}\left(\mathbb{R}^{N+1}\right)$, we denote by $\mathcal{M}^{+}(F)$ the set of nonnegative Radon measures supported on $F$. For any $a>0$, we define

$$
\mathcal{C}_{a}(F)=\sup \left\{\mu(F): \mu \in \mathcal{M}^{+}(F), \text { and } \mathrm{G}_{a} * \mu(z)=\int \mathrm{G}_{a}(z, \zeta) d \mu(\zeta) \leq 1 \forall z \in S\right\} .
$$

The following properties holds true (see e.g. [18, Chapter 1, Section 2]):

(i) $\mathcal{C}_{a}(F)<\infty$ for any $F \in \mathcal{F}\left(\mathbb{R}^{N+1}\right)$;

(ii) if $F_{1}, F_{2} \in \mathcal{F}\left(\mathbb{R}^{N+1}\right)$ with $F_{1} \subseteq F_{2}$, then $\mathcal{C}_{a}\left(F_{1}\right) \leq \mathcal{C}_{a}\left(F_{2}\right)$;

(iii) for every $F \in \mathcal{F}\left(\mathbb{R}^{N+1}\right)$ there exists $\mu^{F}=\mu_{a}^{F} \in \mathcal{M}^{+}(F)$ with $\mathrm{G}_{a} * \mu^{F} \leq 1$ in $\mathbb{R}^{N+1}$ such that

$$
\mu^{F}(F)=\mathcal{C}_{a}(F)
$$

(iv) if $F \subseteq \cup_{k \in \mathbb{N}} F_{k}$ with $F, F_{k} \in \mathcal{F}\left(\mathbb{R}^{N+1}\right)$, then $\mathcal{C}_{a}(F) \leq \sum_{k \in \mathbb{N}} \mathcal{C}_{a}\left(F_{k}\right)$.

The measure $\mu_{a}^{F}$ is called $\mathrm{G}_{a}$-equilibrium measure of $F$, and the function $\mathrm{G}_{a} * \mu^{F}$ is called a $\mathrm{G}_{a}$-equilibrium potential of $F$. Moreover, if $a \leq b$, we have $\mathrm{G}_{b} \leq \mathrm{G}_{a}$ and thus

$$
\mathcal{C}_{a}(F) \leq \mathcal{C}_{b}(F) \quad \forall F \in \mathcal{F}\left(\mathbb{R}^{N+1}\right) .
$$

\section{Proof of the main Results}

Fix a bounded open set $\Omega$ compactly contained in $S$, and let $a_{0} \leq b_{0}$ be the positive constants in 2.3. In this section we finally prove Theorem 1.1, Corollary 1.2 , and Corollary 1.3. Theorem 1.1 consists of two parts, and it deals with the relation between the regularity of $z_{0}=\left(x_{0}, t_{0}\right) \in \partial \Omega$ and the behavior of the Gaussian capacities of the compact sets

$$
\begin{aligned}
\Omega_{k}^{h}\left(z_{0}, \lambda\right)= & \left\{\zeta=(\xi, \tau) \in S \backslash \Omega: \lambda^{k+1} \leq t_{0}-\tau \leq \lambda^{k},\right. \\
& \left.\left(\frac{1}{\lambda}\right)^{h-1} \leq \exp \left(\frac{d^{2}\left(x_{0}, \xi\right)}{t_{0}-\tau}\right) \leq\left(\frac{1}{\lambda}\right)^{h}, \hat{d}\left(z_{0}, \zeta\right) \leq \sqrt{\lambda}\right\}
\end{aligned}
$$


for $h, k \in \mathbb{N}$. For part $(i)$ (concerning the sufficient condition for the regularity), we go through the approach outlined in [30] where we exploit suitable estimates of the Green kernels of subSection 2.2. For part (ii) (the necessary condition), we need to implement a completely new strategy with respect to [30].

4.1. Proof of part (ii). General potential theory provides the link between the regularity and the divergence of a certain series of balayage functions (see, e.g., 31, Proposition 4.12]). In the case of smooth coefficients, one can then use the representation of the balayage as Riesz-potential (namely the convolution of $\Gamma$ with the relevant Riesz measure). The Gaussian estimates for $\Gamma$ give then the divergence of a series of the type (1.3). This strategy has been followed in 30. Here we deal with non-divergence operators with non-smooth coefficients: a new difficulty arises in trying to follow the same approach, due to the lack of suitable Riesz-type representations for $\mathcal{H}$-superharmonic functions. To avoid this problem, we shall make use of the Riesz measures of a prototype (smooth) operator of the type $\frac{1}{\beta} \sum X_{j}^{2}-\partial_{t}$.

Let us then take

$$
\beta=\frac{b_{0}}{a_{0}} \geq 1, \quad \text { and denote } \quad \mathcal{H}^{\beta}=\frac{1}{\beta} \sum_{j=1}^{m} X_{j}^{2}-\partial_{t} .
$$

The operator $\mathcal{H}^{1}=\sum X_{j}^{2}-\partial_{t}$ falls in the class of operators $\mathcal{H}$ we are considering (since $\Lambda \geq 1$ ). Therefore its fundamental solution $\Gamma^{1}$ satisfies the Gaussian estimates 2.3 in $S$. It is easy to see that the fundamental solution of $\mathcal{H}^{\beta}$, which we are going to denote by $\Gamma^{\beta}$, is a rescaled version of $\Gamma^{1}$, i.e.

$$
\Gamma^{\beta}((x, t),(\xi, \tau))=\Gamma^{1}\left(\left(x, \frac{1}{\beta} t\right),\left(\xi, \frac{1}{\beta} \tau\right)\right) .
$$

For all $\left.z=(x, t), \zeta=(\xi, \tau) \in S^{\beta}:=\mathbb{R}^{N} \times\right] \beta T_{1}, \beta T_{2}[\supseteq S$, we have then

$$
\frac{1}{C_{0}} \mathrm{G}_{b_{0}}\left(\left(x, \frac{1}{\beta} t\right),\left(\xi, \frac{1}{\beta} \tau\right)\right) \leq \Gamma^{\beta}(z, \zeta) \leq C_{0} \mathrm{G}_{a_{0}}\left(\left(x, \frac{1}{\beta} t\right),\left(\xi, \frac{1}{\beta} \tau\right)\right) .
$$

From the definitions of $d$-Gaussians and since $\beta \geq 1$, we can recognize that

$$
\begin{aligned}
& \mathrm{G}_{b_{0}}\left(\left(x, \frac{1}{\beta} t\right),\left(\xi, \frac{1}{\beta} \tau\right)\right) \geq \mathrm{G}_{b_{0} \beta}(z, \zeta), \quad \text { and } \\
& \mathrm{G}_{a_{0}}\left(\left(x, \frac{1}{\beta} t\right),\left(\xi, \frac{1}{\beta} \tau\right)\right) \leq c_{d} \beta^{\frac{Q}{2}} \mathrm{G}_{a_{0} \beta}(z, \zeta),
\end{aligned}
$$

where $c_{d}$ is the doubling constant and $Q$ is the relative homogeneous dimension. Hence we get

$$
\frac{1}{C_{0}} \mathrm{G}_{\frac{b_{0}^{2}}{a_{0}}}(z, \zeta) \leq \Gamma^{\beta}(z, \zeta) \leq C_{0} c_{d} \beta^{\frac{Q}{2}} \mathrm{G}_{b_{0}}(z, \zeta) \leq C_{0}^{2} c_{d} \beta^{\frac{Q}{2}} \Gamma(z, \zeta), \quad \forall z, \zeta \in S .
$$

Thus, the fundamental solution of the prototype operator $\mathcal{H}^{\beta}$ is (up to a structural constant) below all the fundamental solutions $\Gamma$ of the operators $\mathcal{H}$ in our class, and it lies above a $d$-Gaussian with exponent $\frac{b_{0}^{2}}{a_{0}}$. Our aim is to prove part (ii) exactly with the choice

$$
\beta_{0}=\frac{b_{0}^{2}}{a_{0}} \geq b_{0} .
$$


We are going to argue by contradiction. So, for a fixed $z_{0} \in \partial \Omega$ and $\lambda \in(0,1)$, suppose that

$$
\sum_{h, k=1}^{+\infty} \frac{\mathcal{C}_{b}\left(\Omega_{k}^{h}\left(z_{0}, \lambda\right)\right)}{\left|B\left(x_{0}, \sqrt{\lambda^{k}}\right)\right|} \lambda^{a h}<+\infty
$$

for some $b \geq \beta_{0}$ and $0<a \leq a_{0}$. We want to exploit this in order to find a nonnegative $\mathcal{H}$-hyperharmonic function $u$ on a suitable neighborhood of $z_{0}$ such that

$$
\liminf _{(S \backslash \Omega) \cap\left\{t<t_{0}\right\} \ni z \rightarrow z_{0}} u(z)>u\left(z_{0}\right) .
$$

We will recall that this fact implies the $\mathcal{H}$-thinness of $(S \backslash \Omega) \cap\left\{t<t_{0}\right\}$ at $z_{0}$, and therefore the non-regularity of $z_{0}$. A similar strategy of proof has been used by Lanconelli in [27, Teorema 3.3], where he compared regularity criteria with respect to two different classical parabolic operators in terms of the difference of their quadratic forms. Here we construct the desired $\mathcal{H}$-hyperharmonic function $u$ with a superposition of Riesz-potentials of the type $\Gamma * \mu^{\beta}$, where $\mu^{\beta}$ are Riesz measures of suitable $\mathcal{H}^{\beta}$-balayage functions.

Let us see the proof in details. For any $h, k \in \mathbb{N}$, there exists a compact set $E_{k}^{h} \subset S$ with the following properties

$$
\left\{\begin{array}{l}
\Omega_{k}^{h}\left(z_{0}, \lambda\right) \subset \operatorname{int}\left(E_{k}^{h}\right), \\
\mathcal{C}_{\beta_{0}}\left(E_{k}^{h}\right) \leq \mathcal{C}_{\beta_{0}}\left(\Omega_{k}^{h}\left(z_{0}, \lambda\right)\right)+\lambda^{k Q}, \\
\sup _{\zeta \in E_{k}^{h}} \mathrm{G}_{a_{0}}\left(z_{0}, \zeta\right) \leq C_{\lambda} \frac{\lambda^{a_{0} h}}{\left|B\left(x_{0}, \sqrt{\lambda^{k}}\right)\right|}, \quad \text { for some positive } C_{\lambda} \text { indipendent of } h, k .
\end{array}\right.
$$

The constant $C_{\lambda}$ might depend on $\lambda, z_{0}$, and structural quantities $\left(a_{0}, Q, \ldots\right)$. One can in fact make the choice

$$
E_{k}^{h}=\left(\Omega_{k}^{h}\left(z_{0}, \lambda\right)\right)_{\frac{1}{m}}:=\left\{\zeta \in S: \exists \bar{\zeta} \in \Omega_{k}^{h}\left(z_{0}, \lambda\right) \text { with } \hat{d}(\zeta, \bar{\zeta}) \leq \frac{1}{m}\right\}
$$

for some $m=m(h, k, \lambda)$. The fact that $\Omega_{k}^{h}\left(z_{0}, \lambda\right) \subset \operatorname{int}\left(E_{k}^{h}\right)$ is trivial with this choice. Recalling that

$$
\sup _{\zeta \in \Omega_{k}^{h}\left(z_{0}, \lambda\right)} \mathrm{G}_{a_{0}}\left(z_{0}, \zeta\right) \leq \frac{\lambda^{a_{0}(h-1)}}{\left|B\left(x_{0}, \sqrt{\lambda^{k+1}}\right)\right|} \leq \frac{c_{d}}{\lambda^{a_{0}+\frac{Q}{2}}} \frac{\lambda^{a_{0} h}}{\left|B\left(x_{0}, \sqrt{\lambda^{k}}\right)\right|},
$$

the third required property can be proved in a straightforward way from the definition of $E_{k}^{h}$ for all $m$ big enough (i.e. by increasing suitably the constant $\frac{c_{d}}{\lambda^{a_{0}+\frac{Q}{2}}}$, for $m$ bigger than some $\left.m_{1}(h, k, \lambda)\right)$. On the other hand, the second property follows from the fact that

$$
\mathcal{C}_{\beta_{0}}\left(\Omega_{k}^{h}\left(z_{0}, \lambda\right)\right)=\inf _{m} \mathcal{C}_{\beta_{0}}\left(\left(\Omega_{k}^{h}\left(z_{0}, \lambda\right)\right)_{\frac{1}{m}}\right)
$$

which holds true since $\Omega_{k}^{h}\left(z_{0}, \lambda\right)=\bigcap_{m}\left(\Omega_{k}^{h}\left(z_{0}, \lambda\right)\right)_{\frac{1}{m}}$

(see [18, Remark at pg. 155]). Once we have established the existence of $E_{k}^{h}$, we can consider the $\mathcal{H}^{\beta}$-balayage $V_{h, k}^{\beta}:=\widehat{R}_{1}^{E_{k}^{h}}$. Since $\mathcal{H}^{\beta}$ has smooth coefficients, by 31, Proposition 8.3] we can represent $V_{h, k}^{\beta}$ as the Riesz potential $\Gamma^{\beta} * \mu_{h, k}^{\beta}$ almost everywhere in $S$, for some nonnegative Radon measure $\mu_{h, k}^{\beta}$ supported in $E_{k}^{h}$. Moreover we have

$$
V_{h, k}^{\beta}=\Gamma^{\beta} * \mu_{h, k}^{\beta} \equiv 1 \quad \text { in } \operatorname{int}\left(E_{k}^{h}\right) \supset \Omega_{k}^{h}\left(z_{0}, \lambda\right) .
$$


We can now define, for $l \in \mathbb{N}$, the function $u_{l}: S \rightarrow[0,+\infty]$ as

$$
u_{l}=\sum_{k=2 l}^{+\infty} \sum_{h=1}^{+\infty} \Gamma * \mu_{h, k}^{\beta} .
$$

By (3.1) we know that $u_{l} \in \mathrm{H}^{*}(S)$. We have also

$$
\left(\overline{\hat{B}\left(z_{0}, \lambda^{l}\right)} \backslash \Omega\right) \cap\left\{t<t_{0}\right\} \subseteq \bigcup_{k=2 l}^{+\infty} \bigcup_{h=1}^{+\infty} \Omega_{k}^{h}\left(z_{0}, \lambda\right) .
$$

Hence, for every $z \in\left(\overline{\hat{B}\left(z_{0}, \lambda^{l}\right)} \backslash \Omega\right) \cap\left\{t<t_{0}\right\}$, we can consider $\bar{k}, \bar{h}$ such that $z \in \Omega_{\bar{k}}^{\bar{h}}\left(z_{0}, \lambda\right)$, and we get

$$
u_{l}(z) \geq \Gamma * \mu_{\bar{h}, \bar{k}}^{\beta}(z) \geq \frac{1}{c_{d} C_{0}^{2} \beta^{\frac{Q}{2}}} \Gamma^{\beta} * \mu_{\bar{h}, \bar{k}}^{\beta}(z)=\frac{1}{c_{d} C_{0}^{2} \beta^{\frac{Q}{2}}}
$$

by 4.2 and 4.6 . On the other hand, we claim that

$$
\lim _{l \rightarrow+\infty} u_{l}\left(z_{0}\right)=0
$$

In fact, by 2.3 and 4.5 we have

$$
\begin{aligned}
u_{l}\left(z_{0}\right) & \leq \sum_{k=2 l}^{+\infty} \sum_{h=1}^{+\infty} \mu_{h, k}^{\beta}\left(E_{k}^{h}\right) \sup _{\zeta \in E_{k}^{h}} \Gamma\left(z_{0}, \zeta\right) \leq C_{0} \sum_{k=2 l}^{+\infty} \sum_{h=1}^{+\infty} \mu_{h, k}^{\beta}\left(E_{k}^{h}\right) \sup _{\zeta \in E_{k}^{h}} \mathrm{G}_{a_{0}}\left(z_{0}, \zeta\right) \\
& \leq C_{0} C_{\lambda} \sum_{k=2 l}^{+\infty} \sum_{h=1}^{+\infty} \mu_{h, k}^{\beta}\left(E_{k}^{h}\right) \frac{\lambda^{a_{0} h}}{\left|B\left(x_{0}, \sqrt{\lambda^{k}}\right)\right|} .
\end{aligned}
$$

Furthermore, by (4.2) and [30, Corollary 2.4] we have $\mu_{h, k}^{\beta}\left(E_{k}^{h}\right)=: \mathcal{C}_{\mathcal{H}^{\beta}}\left(E_{k}^{h}\right) \leq c \mathcal{C}_{\beta_{0}}\left(E_{k}^{h}\right)$ for some structural positive constant $c$. Therefore, by using 4.5 and the doubling condition, we get

$$
\begin{aligned}
u_{l}\left(z_{0}\right) & \leq C_{0} C_{\lambda} c \sum_{k=2 l}^{+\infty} \sum_{h=1}^{+\infty} \mathcal{C}_{\beta_{0}}\left(E_{k}^{h}\right) \frac{\lambda^{a_{0} h}}{\left|B\left(x_{0}, \sqrt{\lambda^{k}}\right)\right|} \\
& \leq C_{0} C_{\lambda} c \sum_{k=2 l}^{+\infty} \sum_{h=1}^{+\infty}\left(\mathcal{C}_{\beta_{0}}\left(\Omega_{k}^{h}\left(z_{0}, \lambda\right)\right)+\lambda^{k Q}\right) \frac{\lambda^{a_{0} h}}{\left|B\left(x_{0}, \sqrt{\lambda^{k}}\right)\right|} \\
& \leq C_{0} C_{\lambda} c \sum_{k=2 l}^{+\infty} \sum_{h=1}^{+\infty} \mathcal{C}_{\beta_{0}}\left(\Omega_{k}^{h}\left(z_{0}, \lambda\right)\right) \frac{\lambda^{a_{0} h}}{\left|B\left(x_{0}, \sqrt{\lambda^{k}}\right)\right|}+\frac{c_{d} C_{0} C_{\lambda} c}{\left|B\left(x_{0}, 1\right)\right|} \sum_{k=2 l}^{+\infty} \sum_{h=1}^{+\infty} \lambda^{k \frac{Q}{2}} \lambda^{a_{0} h}
\end{aligned}
$$

We recall that $b \geq \beta_{0}$ and $0<a \leq a_{0}$, and also $\mathcal{C}_{\beta_{0}} \leq \mathcal{C}_{b}$ by 3.5 . Thus we have

$$
u_{l}\left(z_{0}\right) \leq C_{0} C_{\lambda} c \sum_{k=2 l}^{+\infty} \sum_{h=1}^{+\infty} \mathcal{C}_{b}\left(\Omega_{k}^{h}\left(z_{0}, \lambda\right)\right) \frac{\lambda^{a h}}{\left|B\left(x_{0}, \sqrt{\lambda^{k}}\right)\right|}+\frac{c_{d} C_{0} C_{\lambda} c}{\left|B\left(x_{0}, 1\right)\right|} \sum_{k=2 l}^{+\infty} \sum_{h=1}^{+\infty} \lambda^{k \frac{Q}{2}} \lambda^{a_{0} h} .
$$

The double series at the r.h.s. are both convergent: the second one just because $\lambda<1$, whereas the convergence of the first is our assumption 4.3). The claim is then proved, since 
we can make the r.h.s. as small as we want for large $l$. Thus, we can take $l_{0} \in \mathbb{N}$ such that

$$
u_{l_{0}}\left(z_{0}\right)<\frac{1}{c_{d} C_{0}^{2} \beta^{\frac{Q}{2}}} .
$$

Hence, we deduce from 4.7 that

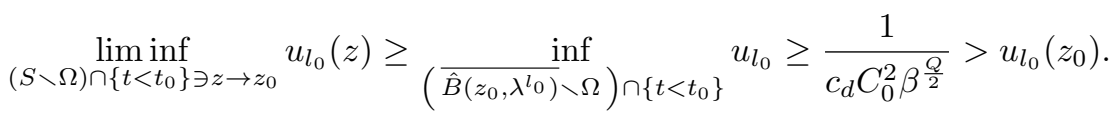

The nonnegative $\mathcal{H}$-hyperharmonic function $u_{l_{0}}$ does the job we were looking for in (4.4). On the other hand, if we put $s=c_{d} C_{0}^{2} \beta^{\frac{Q}{2}}$, we can consider the nonnegative $\mathcal{H}$-hyperharmonic function $v$ defined by

$$
v(z)=s \cdot u_{l_{0}}(z) .
$$

We have by 4.7 that $v \geq 1$ in $\left(\hat{B}\left(z_{0}, \lambda^{l_{0}}\right) \backslash \Omega\right) \cap\left\{t<t_{0}\right\}=\left((S \backslash \Omega) \cap\left\{t<t_{0}\right\}\right) \cap$ $\hat{B}\left(z_{0}, \lambda^{l_{0}}\right)$. Therefore

$$
\widehat{R}_{1}^{\left((S \backslash \Omega) \cap\left\{t<t_{0}\right\}\right) \cap \hat{B}\left(z_{0}, \lambda^{\left.l_{0}\right)}\right.}\left(z_{0}\right) \leq R_{1}^{\left((S \backslash \Omega) \cap\left\{t<t_{0}\right\}\right) \cap \hat{B}\left(z_{0}, \lambda^{l_{0}}\right)}\left(z_{0}\right) \leq v\left(z_{0}\right)<1,
$$

which means by definition that $(S \backslash \Omega) \cap\left\{t<t_{0}\right\}$ is $\mathcal{H}$-thin at $z_{0}$. This fact, together with (3.3) and (3.4), is telling us that $z_{0}$ is not $\mathcal{H}$-regular for $\Omega$. This completes the proof of part (ii).

4.2. Proof of part (i), and corollaries. To prove part (i) we first estimate the modulus of continuity of the PW-solution in terms of a Wiener function, which is then estimated with a series involving capacitary terms. Although this approach is rather delicate, we have the possibility to follow the quantitative proof in 30. We summarize the needed estimate in the following proposition. We first fix some notations. Let us introduce the function

$$
S_{a}^{b}\left(\lambda ; z_{0}, z\right)=\sum_{\mathbb{N} \ni k \leq \frac{\log \hat{d}^{2}\left(z_{0}, z\right)}{\log \lambda}} \sum_{h=1}^{+\infty} \frac{\mathcal{C}_{a}\left(\Omega_{k}^{h}\left(z_{0}, \lambda\right)\right)}{\left|B\left(x_{0}, \sqrt{\lambda^{k}}\right)\right|} \lambda^{b h},
$$

which encodes the behavior of the Wiener type series in $(1.2)$. Moreover, for $l \in \mathbb{N}$ we can consider the compact sets $F_{l}=\left(\overline{\hat{B}\left(z_{0}, \lambda^{\frac{l}{2}}\right)} \cap\left\{t \leq t_{0}\right\}\right) \backslash \Omega$, and the following $\mathcal{H}$-Wiener function for $\Omega$ at $z_{0}$

$$
\left.\mathcal{W}_{\rho}=\sum_{l=1}^{\infty} \rho^{l}\left(1-\widehat{R}_{1}^{F_{l}}\right), \quad \text { for } \rho \in\right] 0,1[
$$

which concerns the behavior of the $\mathcal{H}$-balayage functions around $z_{0}$. Let us also put, for $\sigma>0$,

$$
w_{\rho}(\sigma)=\inf \left\{\mathcal{W}_{\rho}(z): z \in \bar{\Omega}, \hat{d}\left(z_{0}, z\right) \geq \sigma\right\} .
$$

Finally, we denote the oscillation of a function $\varphi \in C(\partial \Omega, \mathbb{R})$ at a point $z_{0} \in \partial \Omega$ and scale $r$ as

$$
\omega_{\varphi}\left(z_{0}, r\right)=\sup _{\hat{d}\left(z, z_{0}\right) \leq r}\left|\varphi(z)-\varphi\left(z_{0}\right)\right| .
$$


Proposition 4.1. Let $z_{0} \in \partial \Omega$ and $\left.\lambda \in\right] 0,1\left[\right.$. For every $0<a \leq a_{0}$ and $b>b_{0}$ there exist positive constants $C$ and $\rho_{0}$ (only depending on $a, b, \lambda$ ) such that

$$
\left|H_{\varphi}^{\Omega}(z)-\varphi\left(z_{0}\right)\right| \leq \inf _{\sigma>0}\left(\omega_{\varphi}\left(z_{0}, \sigma\right)+C \frac{\omega_{\varphi}\left(z_{0}, \operatorname{diam}_{\hat{d}}(\partial \Omega)\right)}{w_{\rho_{0}(\sigma)}} \exp \left(-\frac{1}{C} S_{a}^{b}\left(\lambda ; z_{0}, z\right)\right)\right)
$$

for every $z \in \Omega$, and for all $\varphi \in C(\partial \Omega, \mathbb{R})$.

Proof. Fix $z_{0} \in \partial \Omega$, and $\varphi \in C(\partial \Omega, \mathbb{R})$. We can proceed verbatim as in the proof of 31, Theorem 5.2] and we can get

$$
\left|H_{\varphi}^{\Omega}(z)-\varphi\left(z_{0}\right)\right| \leq \inf _{\sigma>0}\left(\omega_{\varphi}\left(z_{0}, \sigma\right)+\omega_{\varphi}\left(z_{0}, \operatorname{diam}_{\hat{d}}(\partial \Omega)\right) \frac{\mathcal{W}_{\rho}(z)}{w_{\rho}(\sigma)}\right)
$$

for all $z \in \Omega$, and for any $\rho \in] 0,1\left[\right.$. We also notice that $w_{\rho}(\sigma)$ has to be strictly positive by arguing as in [31, inequality (5.6)]. Fix now $\lambda, a$ and $b$ as in the statement. The inequality (4.8) then follows if we can prove the existence of $C$ and $\rho_{0}$ such that

$$
\mathcal{W}_{\rho_{0}}(z) \leq C \exp \left(-\frac{1}{C} S_{a}^{b}\left(\lambda ; z_{0}, z\right)\right) \quad \text { for every } z \in S,
$$

which is the analogous of [30, Theorem 1.2]. We recall here the main steps in the proof, and we refer the reader to [30] for everything else. We denote

$$
\begin{gathered}
D_{k}^{h}\left(z_{0}, \lambda\right)=\bigcup_{j=1}^{h} \Omega_{k}^{j}\left(z_{0}, \lambda\right) \quad \text { for } h, k \in \mathbb{N}, \text { and } \\
Z_{a}^{b}\left(\lambda ; z_{0}, z\right)=\sum_{\mathbb{N} \ni k \leq \frac{\log \hat{d}^{2}\left(z_{0}, z\right)}{\log \lambda} \sum_{h=1}^{+\infty} \frac{\mathcal{C}_{a}\left(D_{k}^{h}\left(z_{0}, \lambda\right)\right)}{\left|B\left(x_{0}, \sqrt{\lambda^{k}}\right)\right|} \lambda^{b h} .}
\end{gathered}
$$

Since $S_{a}^{b}\left(\lambda ; z_{0}, z\right)$ and $Z_{a}^{b}\left(\lambda ; z_{0}, z\right)$ have equivalent behavior and $Z_{a}^{b}\left(\lambda_{1} ; z_{0}, z\right)$ is equivalent to $Z_{a}^{b}\left(\lambda_{2} ; z_{0}, z\right)$ for $\lambda_{1} \neq \lambda_{2}$ by [30, Remark 4.1 and Remark 4.2], it is enough to prove that, if $\lambda>0$ is small enough (less than a structural $\lambda_{0}$ ), then there exist $C$ and $\rho_{0}$ such that

$$
\mathcal{W}_{\rho_{0}}(z) \leq C \exp \left(-\frac{1}{C} Z_{a}^{b}\left(\lambda ; z_{0}, z\right)\right) \quad \text { for every } z \in S .
$$

To this aim, the crucial part of the argument is to prove that there exists a positive constant $C_{1}$ such that

$$
1-\widehat{R}_{1}^{F_{l}}(z) \leq \exp \left(C_{1} \cdot l\right) \exp \left(-\frac{1}{C_{1}} Z_{a}^{b}\left(\lambda ; z_{0}, z\right)\right) \quad \forall z \in S, \text { and for every } l \in \mathbb{N}:
$$

this fact, together with the choice $0<\rho<e^{-C_{1}}$, would imply

$$
\mathcal{W}_{\rho}(z)=\sum_{l=1}^{+\infty} \rho^{l}\left(1-\widehat{R}_{1}^{F_{l}}(z)\right) \leq \exp \left(-\frac{1}{C_{1}} Z_{a}^{b}\left(\lambda ; z_{0}, z\right)\right) \sum_{l=1}^{+\infty}\left(\rho e_{1}^{C}\right)^{l}
$$

and would complete the proof. The proof of 4.10 requires the estimate in 30, Lemma 3.4], which is the most delicate part. We can derive such result in the same exact way. We just mention here that this estimate links the behavior of the $\mathcal{H}$-balayage of a finite union of compact sets $K_{i}$ approaching $z_{0}$ from below with the behavior at $z_{0}$ of certain $G$-equilibrium potentials of $K_{i}$. These equilibrium potentials are defined as the convolution of the Green 
function of subSection 2.2 related to the cylinder $C_{z_{0}}(M, r)$ defined in 2.8 with the measure $\nu_{i} \in \mathcal{M}^{+}\left(K_{i}\right)$ for which

$$
\nu_{i}\left(K_{i}\right)=\sup \left\{\mu\left(K_{i}\right): \mu \in \mathcal{M}^{+}\left(K_{i}\right), \text { and } \int G\left(z_{0}, M, r ; z, \zeta\right) d \mu(\zeta) \leq 1 \forall z \in S\right\} .
$$

The measure $\nu_{i}$ is a (so called) equilibrium measure of $K_{i}$ related to the kernel $G\left(z_{0}, M, r ; \cdot, \cdot\right)$ (see, e.g., [18, 30 ). The Gaussian bounds for the Green functions and the Hölder continuity for the solutions to $\mathcal{H}$ (which in our case comes from the Harnack inequality in 8 , Theorem 15.1]) are the main ingredients in order to get the correct dependence with respect to $r, M$ and, more in general, the proof of the desired estimate.

The proof of part $(i)$ in Theorem 1.1 easily follows from the last proposition. We have just to observe that the hypothesis 1.2 says that $S_{a}^{b}\left(\lambda ; z_{0}, z\right) \rightarrow+\infty$ as $\hat{d}\left(z_{0}, z\right) \rightarrow 0^{+}$. Thus, the right hand side of (4.9) can be made as small as we want for some $\sigma>0$, for any continuous function $\varphi$. Hence $z_{0}$ has to be $\mathcal{H}$-regular, and the proof is complete.

Let us now recall here for clarity the definition of $d$-cone condition (already appeared with $(2.5)$ ).

Definition 4.2. We say that $\Omega$ satisfies the exterior $d$-cone condition at a point $z_{0}=$ $\left(x_{0}, t_{0}\right) \in \partial \Omega$ if there exist $M_{0}, r_{0}, \theta>0$ such that

$$
\left|\left\{x \in \overline{B\left(x_{0}, M_{0} r\right)}:\left(x, t_{0}-r^{2}\right) \notin \Omega\right\}\right| \geq \theta\left|B\left(x_{0}, M_{0} r\right)\right| \quad \text { for every } 0<r \leq r_{0} .
$$

Under this condition it is possible to give a further estimate of the $\mathcal{H}$-Wiener function $\mathcal{W}_{\rho_{0}}$, where $\rho_{0}$ is the constant in Proposition 4.1 .

Proposition 4.3. Assume $\Omega$ satisfies the exterior $d$-cone condition at $z_{0} \in \partial \Omega$. Then there exist $c$ and $\bar{\alpha}$ such that

$$
\mathcal{W}_{\rho_{0}}(z) \leq c\left(\hat{d}\left(z_{0}, z\right)\right)^{\bar{\alpha}} \quad \text { for every } z \in S .
$$

The proof can be done by arguing verbatim as in [30, Theorem 5.4], by using the integral bound in [30, Theorem 1.3]. The last proposition, together with (4.9), says in particular that the $d$-cone condition ensures the $\mathcal{H}$-regularity of $z_{0}$ (see also the recent results in [21, 22] for cone-type criteria for some classes of hypoelliptic operators). We recall that, if $\Omega$ is a cylinder of the type $A \times] t_{1}, t_{2}$ [, the $d$-cone condition is implied by the density condition (2.6) for the set $A \subset \mathbb{R}^{N}$ (see the results in [43, 41] for the boundary regularity of some stationary operators under condition (2.6)).

To complete the proof of Corollary 1.2 , we want also to deduce the hölder regularity of $H_{\varphi}^{\Omega}$ at $z_{0}$ by assuming more control on the oscillation $\omega_{\varphi}\left(z_{0}, r\right)$. This is again a consequence of Proposition 4.3 and 4.9 (see [30, Theorem 1.4] and [31, Proposition 5.7] for more details).

Finally, we are just left with the proof of Corollary 1.3

Proof of Corollary 1.3. Let $E$ be the subset of $\partial \Omega$ as in the statement. For the case $f \equiv 0$, we have proved with Theorem 1.1 that the choice $u=H_{\varphi}^{\Omega}$ solves the problem, for any continuous datum $\varphi$ on $\partial \Omega$.

On the other hand, let us consider the case of a function $f \in \mathcal{C}_{X}^{\beta}$ in a neighborhood $\Omega_{0}$ of $\bar{\Omega}$. Let $\psi \in C_{0}^{\infty}\left(\Omega_{0}\right)$ be a cut-off function such that $\psi=1$ in $\bar{\Omega}$. Then $\widetilde{f}=f \psi \in \mathcal{C}_{X}^{\beta}\left(\mathbb{R}^{N+1}\right)$. 
We set $v(x, t)=-\int_{\mathbb{R}^{N} \times\left[T_{0}, t\right]} \Gamma(x, t ; \zeta) \widetilde{f}(\zeta) d \zeta$, where $T_{0}$ is a fixed time "below" $\Omega_{0}$. In this way $v \in \mathcal{C}_{X}^{2}\left(\mathbb{R}^{N} \times\right] T_{0}, T_{2}\left[\right.$ ) and $\mathcal{H} v=\tilde{f}$ in $\left.\mathbb{R}^{N} \times\right] T_{0}, T_{2}$ [ (see [8, Theorem 10.7]). It is now sufficient to choose $u=v+H_{\varphi-v}^{\Omega}$.

\section{REFERENCES}

1. A.A. Agrachev, D. Barilari, U. Boscain, Introduction to Riemannian and sub-Riemannian geometry. (Lecture Notes), http://webusers.imj-prg.fr/ davide.barilari/Notes.php

2. A. Bonfiglioli, E. Lanconelli, F. Uguzzoni, Uniform Gaussian estimates of the fundamental solutions for heat operators on Carnot groups. Adv. Differential Equations 7 (2002) 1153-1192

3. A. Bonfiglioli, E. Lanconelli, F. Uguzzoni, Fundamental solutions for non-divergence form operators on stratified groups. Trans. Amer. Math. Soc. 356 (2003) 2709-2737

4. A. Bonfiglioli, F. Uguzzoni, Maximum principle and propagation for intrinsicly regular solutions of differential inequalities structured on vector fields. J. Math. Anal. Appl. 322 (2006) 886-900

5. A. Bonfiglioli, F. Uguzzoni, Harnack inequality for non-divergence form operators on stratified groups. Trans. Amer. Math. Soc. 359 (2007) 2463-2482

6. J.M. Bony, Principe du maximum, inégalité de Harnack et unicité du probleme de Cauchy pour les opérateurs elliptiques dégénérés, Ann. Inst. Fourier (Grenoble) 19 (1969) 277-304

7. M. Bramanti, L. Brandolini, Schauder estimates for parabolic nondivergence operators of Hörmander type. J. Differential Equations 234 (2007) 177-245

8. M. Bramanti, L. Brandolini, E. Lanconelli, F. Uguzzoni, Non-divergence equations structured on Hörmander vector fields: heat kernels and Harnack inequalities. Mem. Amer. Math. Soc. 204 no. 961 (2010)

9. J.-H Cheng, J.-F Hwang, A. Malchiodi, P. Yang, Minimal surfaces in pseudohermitian geometry, Ann. Sc. Norm. Super. Pisa Cl. Sci. (5) 4 (2005) 129-177

10. C. Cinti, K. Nyström, S. Polidoro, A boundary estimate for non-negative solutions to Kolmogorov operators in non-divergence form, Ann. Mat. Pura Appl. 191 (2012) 1-23

11. C. Cinti, K. Nyström, S. Polidoro, A Carleson-type estimate in Lipschitz type domains for non-negative solutions to Kolmogorov operators, Ann. Sc. Norm. Super. Pisa Cl. Sci. (5) 12 (2013) 439-465

12. C. Constantinescu, A. Cornea, Potential theory on harmonic spaces, Springer-Verlag, Berlin, 1972

13. G. Di Fazio, C.E. Gutiérrez, E. Lanconelli, Covering theorems, inequalities on metric spaces and applications to PDE's. Math. Ann. 341 (2008) 255-291

14. L.C. Evans, R.F. Gariepy, Wiener's criterion for the heat equation. Arch. Rational Mech. Anal. 78 (1982) 293-314

15. E.B. Fabes, N. Garofalo, E. Lanconelli, Wiener's criterion for divergence form parabolic operators with $C^{1}$-Dini continuous coefficients. Duke Math. J. 59 (1989) 191-232

16. B. Franchi, R. Serapioni, F. Serra Cassano, Rectifiability and perimeter in the Heisenberg group, Math. Ann. 321 (2001) 479-531

17. M. Frentz, N. Garofalo, E. Götmark, I. Munive, K. Nyström, Non-divergence form parabolic equations associated with non-commuting vector fields: boundary behavior of nonnegative solutions, Ann. Sc. Norm. Super. Pisa Cl. Sci. (5) 11 (2012) 437-474

18. B. Fuglede, On the theory of potentials in locally compact spaces. Acta Math. 103 (1960) 139-215

19. N. Garofalo, E. Lanconelli, Wiener's criterion for parabolic equations with variable coefficients and its consequences. Trans. Amer. Math. Soc. 308 (1988) 811-836

20. N. Garofalo, F. Segàla, Estimates of the fundamental solution and Wiener's criterion for the heat equation on the Heisenberg group. Indiana Univ. Math. J. 39 (1990) 1155-1196

21. A.E. Kogoj, On the Dirichlet Problem for hypoelliptic evolution equations: Perron-Wiener solution and a cone-type criterion. J. Differential Equations 262 (2017) 1524-1539

22. A.E. Kogoj, E. Lanconelli, G. Tralli, Wiener-Landis criterion for Kolmogorov-type operators, submitted.

23. N.V. Krylov, T. Yastrzhembskiy, On nonequivalence of regular boundary points for second-order elliptic operators. Comm. Partial Differential Equations 424 (2017) 366-387

24. E. Lanconelli, Sul problema di Dirichlet per l'equazione del calore. Ann. Mat. Pura Appl. (4) 97 (1973) 83-114

25. E. Lanconelli, Sul problema di Dirichlet per equazioni paraboliche del secondo ordine a coefficienti discontinui. Ann. Mat. Pura Appl. (4) 106 (1975) 11-38 
26. E. Lanconelli, Sul modulo di continuità alla frontiera della soluzione del problema di Dirichlet relativo ad una classe di operatori parabolici. Ann. Sc. Norm. Super. Pisa Cl. Sci. (4) 2 (1975) 335-358

27. E. Lanconelli, Sul confronto della regolarità dei punti di frontiera rispetto ad operatori lineari parabolici diversi. Ann. Mat. Pura Appl. (4) 114 (1977) 207-227

28. E. Lanconelli, A. Montanari, On a class of fully nonlinear PDEs from complex geometry. In "Recent trends in nonlinear partial differential equations.I. Evolution problems", Contemp. Math. 594 (2013) 231-242

29. E. Lanconelli, A. Pascucci, S. Polidoro, Linear and nonlinear ultraparabolic equations of Kolmogorov type arising in diffusion theory and in finance. In "Nonlinear problems in mathematical physics and related topics,II", Int. Math. Ser. (N. Y.) 2 (2002) 243-265

30. E. Lanconelli, G. Tralli, F. Uguzzoni, Wiener-type tests from a two-sided Gaussian bound. Ann. Mat. Pura Appl. (4) 196 (2017) 217-244

31. E. Lanconelli, F. Uguzzoni, Potential analysis for a class of diffusion equations: a Gaussian bounds approach. J. Differential Equations 248 (2010) 2329-2367

32. E.M. Landis, Necessary and sufficient conditions for the regularity of a boundary point for the Dirichlet problem for the heat equation. Dokl. Akad. Nauk SSSR 185 (1969) 517-520

33. A. Malchiodi, F. Uguzzoni, A perturbation result for the Webster scalar curvature problem on the CR sphere. J. Math. Pures Appl. (9) 81 (2002) 983-997

34. V. Martino, G. Tralli, A Jellett type theorem for the Levi curvature, to appear in J. Math Pures Appl.

35. I. Munive, Boundary behavior of non-negative solutions of the heat equation in sub-Riemannian spaces. Potential Anal. 37 (2012) 333-352

36. A. Nagel, E.M. Stein, S. Wainger, Balls and metrics defined by vector fields I: Basic properties. Acta Math. 155 (1985) 103-147

37. A.A. Novruzov, Certain criteria for the regularity of boundary points for linear and quasilinear parabolic equations. Dokl. Akad. Nauk SSSR 209 (1973) 785-787

38. I. Petrowsky, Zur ersten Randwertaufgabe der Wärmeleitungsgleichung. Compositio Mathematica 1 (1935) 383-419

39. B. Pini, Sulla soluzione generalizzata di Wiener per il primo problema di valori al contorno nel caso parabolico. Rend. Sem. Mat. Univ. Padova 23 (1954) 422-434

40. V. Scornazzani, The Dirichlet problem for the Kolmogorov operator. Boll. Un. Mat. Ital. C (5) 18 (1981) 43-62

41. G. Tralli, F. Uguzzoni, Wiener criterion for X-elliptic operators. J. Differential Equations 259 (2015) 6510-6527

42. F. Uguzzoni, Cone criterion for non-divergence equations modeled on Hörmander vector fields. Lecture Notes of Semin. Interdiscip. di Matematica 6 (2007) 227-241

43. F. Uguzzoni, Estimates of the Green function for X-elliptic operators. Math. Ann. 361 (2015) 169-190

Dipartimento di Matematica, Sapienza Università di Roma, P.le Aldo Moro 5 - 00185 Roma, ITALY.

E-mail address, Corresponding author: tralli@mat.uniroma1.it

Dipartimento di Matematica, Università degli Studi di Bologna, Piazza di Porta S. Donato, 5 - 40126 Bologna, Italy.

E-mail address: francesco.uguzzoni@unibo.it 